ИЗВЕСТИЯ АКАДЕМИИ НАУК ЭСТОНСКОП ССР. ТОМ 30 ФИЗИКА * МАТЕМАТИКА. 1981, № 1

\title{
ДЕЦЕНТРАЛИЗАЦИЯ ЗАДАЧИ УПРАВЛЕНИЯ оБзоР
}

(Представил Н. Алумяэ)

\section{1. Введение}

Автоматизированную систему управления технологическим процессом на стадии проектирования принято рассматривать состоящей из объекта управления и многослойного децентрализованного регулятора $\left[{ }^{1-7}\right]$. Оптимальное управление в таких системах вырабатывается многими центрами управления, каждый из которых действует на основе частичной информации о системе в целом. Первый слой регулятора, как правило, должен поддерживать переменные состояния объекта вблизи заданных значений. Второй слой определяет последние, решая задачу оптимизации в детерминированной постановке. Третий слой уточняет неизвестные значения параметров объекта, используя поступающую о процессе информацию, четвертый и более высокие слои могут уже, например, изменять или уточнять задачи управления нижних слоев по мере изменения требований к технологическому процессу. Каждая задача слоя может, в свою очередь, решаться децентрализованно, совместными усилиями нескольких центров управления, организованных для этой цели. Децентрализация задачи управления первого слоя в основном достигается методами выделения в линеаризованной модели объекта взаимно малосвязанных частей $\left[{ }^{8-11}\right]$, а также методами исследования условий управляемости, наблюдаемости и устойчивости, при которых невзаимосвязанные регуляторы могут работать на взаимосвязанном объекте $\left[{ }^{12-26}\right]$. Фактическая размерность действующих регуляторов первого слоя редко превышает пяти, хотя общее число таких регуляторов на технологическом объекте достигает сотен.

Задача управления второго слоя обычно формулируется в виде задачи динамической оптимизации на нелинейной модели объекта. В данном обзоре, в целях наглядности изложения, используется линейная модель (1.1), которая включает $m$ взаимосвязанных, быть может, пространственно рассредоточенных частей - подсистем,

$$
\dot{x}=A x+\sum_{i=1}^{m} \bar{B}_{i} u_{i},
$$

точнее 


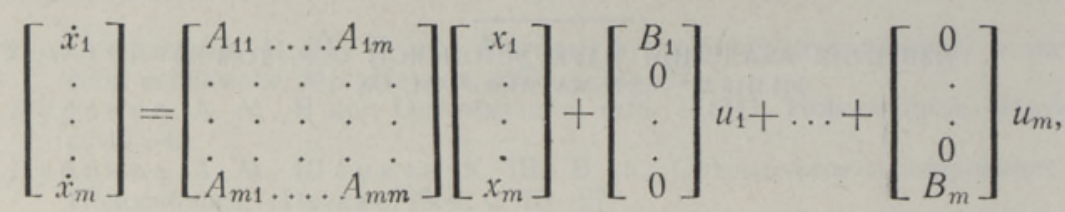

где $x_{i}-n_{i}$-мерный вектор состояния $i$-й подсистемы, $u_{i}-p_{i}$-мерный вектор управления $i$-й подсистемы, $A$ и $\bar{B}_{i}$ - известные числовые матрицы.

Примем также, что качество подсистемы оценивается локальной функцией цели

$$
G_{i}=\frac{1}{2} \int_{0}^{T}\left(x^{\prime} \bar{Q}_{i} x+u_{i}^{\prime} R_{i} u_{i}\right) d t, \quad i=1,2, \ldots, m,
$$

где матрицы $\bar{Q}_{i}=\bar{Q}_{i}^{\prime} \geqslant 0, R_{i}=R_{i}{ }_{i}>0$, штрих означает транспонирование, $\bar{Q}_{i}$ - блочно-диагональная матрица, содержащая один ненулевой блок $Q_{i}$.

Очевидно, минимизация суммы всех локальных функций цели $G=\sum_{i=1}^{m} G_{i}$ по управлениям $u_{i} \in R^{p t}, i=1,2, \ldots, m$, при выполненин условий (1.2) приведет к известным двухуровневым схемам декомпознции-координации $\left[{ }^{27-76}\right]$. Если же каждый локальный регулятор будет стремиться управлять своей частью системы так, чтобы достичь оптимума только своей целевой функцин, и если, кроме того, будет установлен приоритет в последовательности определения $u_{i}$, то решение будет определяться стратегией Стаккельберга (приоритетная подсистема при выборе своего оптимального управления учитывает реакцию последующих систем) $\left[{ }^{77-86}\right]$, или стратегией Нэша (эффект от управления распространяется только на последующие подсистемы, обратного воздействия нет) $\left[{ }^{87-95}\right]$. В последнем случае необходимо, чтобы в уравнении (1.2) $A_{i j}=0$ при $j>i, i=1,2, \ldots, m$.

В указанных трех задачах управления взаимосвязи между подсистемами обусловливаются только взаимосвязями объекта. При $A_{i j}=0$, $j \neq i, i=1,2, \ldots, m$, задача управления сетью подсистем сводится к $m$ несвязанным подзадачам классической оптимизации.

\section{2. Метод декомпозиции-координации в оптимизации сети подсистем}

Примем, что задача управления состоит в определении $u_{i} \in R^{p}$, $i=1,2, \ldots, m$, которые минимизируют сепарабельную функцию цели

$$
G=\sum_{i=1}^{m} G_{i}
$$

при ограничениях $(1.2)$ и заданном $x_{i}(0)$, где $G_{i}$ дается выражением (1.3).

Функция Лагранжа, которая соответствует данной задаче, имеет вид

$$
L \triangleq \sum_{i=1}^{m} \int_{0}^{T}\left[\frac{1}{2}\left(x_{i}^{\prime} Q_{i} x_{i}+u_{i}^{\prime} R_{i} u_{i}\right)+p^{\prime}{ }_{i}\left(-\dot{x}_{i}+A_{i i} x_{i}+B_{i} u_{i}+z_{i}\right)+\right.
$$




$$
\left.+\lambda_{i}^{\prime}\left(z_{i}-\sum_{j \neq i}^{m} A_{i j} x_{j}\right)\right] d t
$$

Учитывая, что

$$
\sum_{i=1}^{m} \lambda_{i}^{\prime} \sum_{j \neq i}^{m} A_{i j} x_{j}=\sum_{i=1}^{m} \sum_{j \neq i}^{m} \lambda_{j}^{\prime} A_{j i} x_{i}
$$

можем записать

$$
L=\sum_{i=1}^{m} L_{i}\left(x_{i}, u_{i}, z_{i}, p_{i}, \lambda_{1}, \ldots, \lambda_{m}\right) .
$$

В данном примере $L$ имеет единственную седловую точку [ $\left.{ }^{30}\right]$. Задача управления эквивалентна задаче минимизации $L_{i}$ по $x_{i}, u_{i}, z_{i}$ и максимизации по переменным $p_{i}$ и $\lambda$, для решения которой органнзуется итерационный процесс $\left[{ }^{27-45}\right]$. Необходимые вычисления на каждом шаге итерации распределяются между центром второго уровня и подсистемами. Вычисления в подсистемах осуществляются независимо друг от друга, объем параллельных вычислений при этом различен и зависит от того, какие переменные из (2.2) фиксированы.

2.1. С хема с координацией функции цели. Очевидно, если фиксировать траекторию $\lambda(t)=\lambda^{l}(t), 0 \leqslant t \leqslant T$, где $l$ означает номер итерации, то все $L_{i}$ будут содержать лишь переменные с индексом $i$, т. е. $L_{i}$ будут соответствовать независимым друг от друга $m$ подзадачам определения оптимальных $u_{i}\left(\lambda^{l}\right), x_{i}\left(\lambda^{l}\right), z_{i}\left(\lambda^{l}\right), \quad p_{i}\left(\lambda^{l}\right) \quad\left[{ }^{41,46,47}\right]$. Частная производная $L$ по $\lambda_{i}$ при $\lambda_{i}=\lambda_{i}{ }^{l}$ равна невязке по взаимосвязям

$$
\varepsilon_{i}^{l}=z_{i}\left(\lambda^{l}\right)-\sum_{j \neq i}^{m} A_{i j} x_{j}\left(\lambda^{l}\right) .
$$

$\mathrm{B}\left[{ }^{43}\right]$ предложен следующий алгоритм уточнения $\lambda(t)$, приводящий $\mathrm{k}$ $L_{\lambda}=0$ :

$$
\begin{aligned}
& \lambda^{l+1}=\lambda^{l}+\alpha^{l} d^{l}, \\
& d^{l}=\beta^{l} d^{l-1}+\varepsilon^{l}, d^{0}=\varepsilon^{0}, \\
& \beta^{l}=\int_{0}^{T}\left(\varepsilon^{\prime} \varepsilon\right)^{l} d t\left(\int_{0}^{T}\left(\varepsilon^{\prime} \varepsilon\right)^{l-1} d t\right)^{-1},
\end{aligned}
$$

а $\alpha^{l}$ подбирается прямым поиском [ $\left.{ }^{30}\right]$. Для обеспечения невырожденности решения по переменной $z_{i}$ к $G_{i}$ добавляется слагаемое $z^{\prime}{ }_{i} D_{i} z_{i}$, $D_{i}=D^{\prime}{ }_{i}>0\left[{ }^{49}\right]$. Схема с координацией функции цели в настоящее время достаточно полно исследована и нашла практическое применение в различных системах управления $[31,34,53-57]$.

2.2. С хем а с координацией модели объекта. Если в $L_{i}$ вектор суммарного воздействия от остальных подсистем $z_{i}$ фиксирован, получим схему с координацией модели $\left[{ }^{28,30,50}\right]$. Фиксирование переменной $z_{i}=z_{i}^{l}$ фиксирует также, согласно модели объекта, все остальные переменные состояния, поэтому всегда $L \equiv G$. Для уточнения $z_{i}^{l}$ используется значение $L_{x}=G_{x}$ при $z_{i}^{l}$ и $x_{i}^{l}$. Очевидно, если $L_{x}=0$, то оптимальное управление найдено.

Схема с координацией модели и схема с координацией функции цели для линейных систем образуют двойственную пару $\left[{ }^{30}\right]$. 
2.3. С ем а к кордин ацией сопряженной переменной $p_{i}$. Эта схема нашла широкое применение благодаря ряду достоннств $\left[{ }^{39}\right]$ : ограничения по взаимосвязям удовлетворяются на каждом шаге итерации (что позволяет использовать приближенно оптимальное управление на каждом шаге итерации), локальные задачи решаются при фиксированной траектории сопряженной переменной (т. е. решения двухточечных краевых задач в подсистемах не требуется).

2.4. Схема с координацией функции цели и модели. Если переменные $\lambda=\lambda^{*}$ и $z_{i}=z_{i}^{*}$ фиксированы, функции Лагранжа $L_{i}\left(u_{i}, x_{i}, p_{i}, z_{i}{ }^{*}, \lambda^{*}\right)$ будет соответствовать задача оптимизации, которая сводится к решению двухточечной краевой задачи

$$
\begin{array}{ll}
\dot{x}_{i}=A_{i i} x_{i}-B_{i} R_{i}^{-1} B^{\prime}{ }_{i} p_{i}+z_{i}^{*}, & x_{i}(0)=x_{i 0}, \\
\dot{p}_{i}=-A^{\prime}{ }_{i i} p_{i}+Q_{i} x_{i}+g_{i}^{*}, & p_{i}(T)=0
\end{array}
$$

при фиксированных $z_{i}$ и $g_{i}, i=1,2, \ldots, m$, где

$$
g_{i}=\sum_{j \neq i}^{m} A^{\prime}{ }_{j i} p_{j} .
$$

Уравнения $L_{z}=0$ и $L_{\lambda}=0$ дают

$$
z_{i}=\sum_{j \neq i}^{m} A_{i j} x_{j}, \quad p_{i}=-\lambda_{i} .
$$

В [46,51,52] предложено вычислять уточненные $z_{i}$ и $\lambda_{i}$ подстановкой в уравнения $L_{z}=0$ и $L_{\lambda}=0$ последних значений $x$ и $p$, полученных в подсистемах, что приводит к простому алгоритму:

$$
\left[\begin{array}{l}
z_{i}^{*} \\
g_{i}^{*}
\end{array}\right]^{l+1}=\left[\begin{array}{c}
\sum_{j \neq i}^{m} A_{i j} x_{j} \\
\sum_{j \neq i}^{m} A^{\prime}{ }_{j i} p_{j}
\end{array}\right]^{l} .
$$

Алгоритм (2.6) предъявляет к центральной ЭВМ весьма скромные требования, так как уточнение $z_{i}{ }^{*}$ и $g_{i}{ }^{*}$ осуществляется фактически простым обменом данными между подсистемами. Условия сходимөсти итерационного процесса выражаются через матрицы $A_{i i}, A_{i j}, B_{i}, R_{i}, Q_{i}$ $\left[{ }^{46}\right]$. Алгоритм (2.6) считается быстросходящимся и его рекомендуется применять для оптимизации переходных процессов в реальном времени $\left[{ }^{48}\right]$.

Решение системы уравнений $(2.3)-(2.5)$ имеет вид $p_{i}=P_{i i} x_{i}+v_{i}$, а оптимальное управление, согласно условию $L_{u}=0$, вид

$$
u_{i}=-R_{i}^{-1} B_{i}^{\prime}\left(P_{i i} x_{i}+v_{i}\right)
$$

где $P_{i i}$ есть решение матричного уравнения Риккатти

$$
\dot{P}_{i i}+P_{i i} A_{i i}+A^{\prime}{ }_{i i} P_{i i}-P_{i i} B_{i} R_{i}^{-1} B_{i}^{\prime} P_{i i}=0, \quad P_{i i}(T)=0,
$$

а $v_{i}$ - решение линейного дифференциального уравнения

$$
\dot{v}_{i}=\left(P_{i i} B_{i} R_{i}^{-1} B_{i}^{\prime}-A^{\prime}{ }_{i i}\right) v_{i}-P_{i i} z_{i}^{*}+g_{i}^{*},-v_{i}(T)=0 .
$$


К вышеописанной двухуровневой схеме (2.3)-(2.6) децентралпзованного решения задачи управления примыкает схема с коррекцней на псевдомодели $\left[{ }^{30,48,51}\right]$, согласно которой задается приближенно оптимальная траектория движения управляемой системы $x^{*}(t), 0 \leqslant t \leqslant T$, и с ее помощью фиксируется суммарное воздействие от остальных подсистем

$$
z_{i}^{*}=\sum_{j \neq i}^{m} A_{i j} x_{j}^{*} .
$$

В уравнение (1.3) добавляется слагаемое $\left(x_{i}-x_{i}{ }^{*}\right)^{\prime} c Q_{i}\left(x_{i}-x_{i}{ }^{*}\right), c \gg 1$. Задачей подсистемы является минимизация локальной модифицированной функции цели $G_{i}$ по $u_{i} \in R^{p t}$ при ограничениях $(2.3)$, в которых $z_{i}{ }^{*}$ определяется выражением $(2.10)$. В [48] приведен алгоритм коррекции $x_{i}{ }^{*}$, использующий сопряженный градиент функции цели $G=$ $=\sum_{i=1}^{m} G_{i}$ относительно $x_{i}{ }^{*}$ для итерационного сближения действительной $x(t)$ и заданной $x_{i}^{*}(t)$ траекторий системы. В $\left[{ }^{44}\right]$ предложено вместо $x_{i}^{*}(t)$ задавать приближенное управление $u_{i}^{*}(t)$.

Различные схемы децентрализованного решения задачи управления методом декомпозиции-координации получили практическое применение в системах управления производством сахара $\left[{ }^{4}\right]$, уличным двнжением $\left[{ }^{68}\right]$, качеством воды в речном бассейне $\left[{ }^{31}\right]$ и в других системах управления $\left[{ }^{53-67}\right]$, а также в задачах статической оптимизации систем большой размерности $\left[{ }^{28,69-76}\right]$.

\section{3. Стратегия Нэша в оптимизации ациклической сети подсистем}

Рассмотрим частный случай системы (1.2), когда матрицы $A_{i j}=0$ при $j>i, i=1,2, \ldots, m$. В этом случае подсистемы $A_{i i}$ взаимосвязаны согласно структуре ациклического графа, т. е. воздействия предшествующих подсистем (в виде решений известных дифференциальных уравнений) учитываются последующими подсистемами, а обратное воздействие отсутствует.

Управление $u_{1} \in R^{p_{1}}$ первой подсистемы

$$
\dot{x}_{1}=A_{11} x_{1}+B_{1} u_{1}
$$

минимизирующее

$$
G_{1}=\int_{0}^{\infty}\left(x_{1}^{\prime} Q_{1} x_{1}+u_{1}^{\prime} R_{1} u_{1}\right) d t
$$

при заданном $x_{1}(0)$, есть линейная функция текущего состояния $u_{1}=$ $=-R_{1}^{-1} B_{1} P_{11} x_{1}$, где $\left(n_{1} \times n_{1}\right)$-матрица $P_{11}$ дается решением алгебраического уравнения Риккатти

$$
P_{i i} A_{i i}+A^{\prime}{ }_{i i} P_{i i}-P_{i i} B_{i} R_{i}^{-1} B^{\prime}{ }_{i} P_{i i}=0 .
$$

Движение первой подсистемы, замкнутой оптимальной локальной обратной связью, теперь описывается уравнением

$$
\dot{x}_{i}=\tilde{A}_{i} x_{i}
$$

где $\widetilde{A}_{i}=\left(A_{i i}-B_{i} R_{i}^{-1} B_{i}^{\prime} P_{i i}\right)$. 
Вторая подсистема должна учитывать воздействие от первой, т. е. для нее будем иметь следующую модель

$$
\left[\begin{array}{c}
\dot{x}_{1} \\
\dot{x}_{2}
\end{array}\right]=\left(\begin{array}{ll}
A_{1} & 0 \\
A_{12} & A_{22}
\end{array}\right)\left[\begin{array}{c}
x_{1} \\
x_{2}
\end{array}\right]+\left[\begin{array}{c}
0 \\
B_{2}
\end{array}\right] u_{2}
$$

и следующую функцию цели

$$
G_{2}=\int_{0}^{\infty}\left(\tilde{x}_{2}^{\prime} \bar{Q}_{2} \tilde{x}_{2}+u_{2}^{\prime} R_{2} u_{2}\right) d t
$$

где $\tilde{x}_{2}=\left(x_{1}^{\prime}, x_{2}^{\prime}\right)^{\prime}$. Оптимальное управление второй подсистемы примет вид

$$
u_{i}^{*}=-R_{i}^{-1} B_{i}^{-} \sum_{j=1}^{i} P_{i j} x_{j}
$$

где $P_{21}$ является решением линейного матричного уравнения

$$
-P_{i j} \tilde{A}_{j}-A^{\prime}{ }_{i} P_{i j}=\sum_{k=j+1}^{i} P_{i k} A_{h j},
$$

а $P_{22}$ - решением уравнения (3.1).

Как показано в $\left[{ }^{93}\right]$, решение (3.4) существует, а замкнутая локальными обратными связями система устойчива. Уравнения (3.1), (3.3), (3.4) позволяют последовательно определять оптимальные управления всех подсистем сети. Нужно отметить, что добавление $(m+1)$-й подсистемы не требует пересчета оптимального управления предшествующих $m$ подсистем.

Очевидно, оптимальные управления подсистем $u_{1}^{*}, \ldots, u_{m}{ }^{*}$ удовлетворяют равновесной стратегии Нэша:

$$
G_{i}\left(u_{1}^{*}, \ldots, u_{i-1}^{*}, u_{i}, u_{i+1}^{*}, \ldots, u_{m}^{*}\right) \geqslant G_{i}\left(u_{1}^{*}, \ldots, u_{m}^{*}\right),
$$

где $u_{i} \in R^{p t}$. В $\left[{ }^{93}\right]$ дано обобщение описанной задачи на нелинейные подсистемы и на более сложные структуры сети, а также приведен пример управления скоростью и расстоянием цепочки движущихся объектов.

Децентрализация задачи управления на основе стратегии Нэша нашла применение при управлении качеством воды в речных бассейнах $\left[{ }^{94}\right]$, при управлении производством электроэнергии $\left[{ }^{95}\right]$ и может быть использована во многих других системах управления, между подсистемами которых имеет место однонаправленное воздействие.

\section{4. Стратегия Стаккельберга в оптимизации сети подсистем}

Пусть дана полностью управляемая система (1.1), в которой действуют $m$ подсистем, каждая из которых стремится минимизировать свой локальный критерий качества (1.3) путем определения $u_{i} \in R^{p t}$ при заданном начальном состоянии $x_{0}$. При этом в оптимальном $u_{i}$ должны быть учтены как воздействие от предшествующих подсистем, так и реакция на $u_{i}$ всех последующих подсистем. Последнее обстоятельство делает задачу управления первой подсистемы наиболее сложной. Понятно также, что добавление $(m+1)$-й подсистемы влечет за собой изменение постановки задачи управления и решение ее заново. Пронллюст- 
рируем сказанное на системе, состоящей из трех подсистем $\left[{ }^{77}\right]$. Необходимые условия оптимальности последней, третьей, подсистемы дают двухточечную краевую задачу:

$$
\begin{array}{ll}
\dot{x}=A x-B_{3} R_{3}^{-1} B^{\prime}{ }_{3} p_{3}+B_{2} u_{2}+B_{1} u_{1}, & x(0)=x_{0}, \\
-\dot{p}_{3}=A^{\prime} p_{3}+\bar{Q}_{3} x, & p_{3}(T)=0 ;
\end{array}
$$

оптимальное управление $u_{3}=-R_{3}{ }^{-1} B^{\prime}{ }_{3} p_{3}$ выражается неявно через $u_{1}$ и $u_{2}$. Необходимые условия оптимальности второй подсистемы приводят к более сложной задаче

$$
\begin{array}{ll}
\dot{x}=A x-S_{3} p_{3}-S_{2} p_{2}+B_{1} u_{1}, & x(0)=x_{0}, \\
-\dot{p}_{2}=A^{\prime} p_{2}+\bar{Q}_{2} x+\bar{Q}_{3} g_{3}, & p_{2}(T)=0, \\
\dot{g}_{3}=S_{3} p_{2}+A g_{3}, & g_{3}(0)=0,
\end{array}
$$

где $S_{i}=B_{i} R_{i}^{-1} B^{\prime}{ }_{i}$, а $p_{3}$ дается уравнением (4.2). Оптимальное управление $u_{2}=-R_{2}{ }^{-1} B^{\prime}{ }_{2} p_{2}$ выражается неявно через $u_{1}$. Наконец, первая подсистема должна определить $u_{1}=-R_{1}{ }^{-1} B^{\prime}{ }_{1} p_{1}$ из следующей системы уравнений:

$$
\begin{array}{rlr}
\dot{x} & =A x-S_{3} p_{3}-S_{2} p_{2}-S_{1} p_{1}, & x(0)=x_{0}, \\
-\dot{p}_{1} & =A^{\prime} p_{1}+\bar{Q}_{1} x+\bar{Q}_{2} g_{1}+\bar{Q}_{3} g_{2}, & p_{1}(T)=0, \\
\dot{g}_{1}=S_{2} p_{1}+A g_{1}+S_{3} g_{\theta}, & g_{1}(0)=0, \\
\dot{g}_{2}=S_{3} p_{1}+A g_{2}, & g_{2}(0)=0, \\
-\dot{g}_{0}=\bar{Q}_{3} g_{1}+A^{\prime} g_{\theta}, & g_{0}(T)=0,
\end{array}
$$

а $p_{3}, p_{2}, g_{3}$ даются уравнениями (4.2), (4.3), (4.4) соответственно.

Данная процедура определяет оптимальное управление как функцню времени и начального состояния. В $\left[{ }^{78}\right]$ приведена процедура синтеза закона замкнутого управления в виде

$$
u_{i}(t)=K_{i}(t) x(t),
$$

а в $\left[{ }^{79,80}\right]$ получен закон управления с обратной связью

$$
u_{i}(t)=K_{i} x(t)
$$

Стратегия Стаккельберга нашла наиболее широкое применение в задачах управления системами, состоящнми только из двух подсистем $\left[{ }^{81-86}\right]$.

\section{5. Заключение}

Рассмотренные три постановки задачи управления второго слоя многослойной системы управления и краткий перечень работ по децентрализованной стабилизации позволили охватить сушественную часть лите. ратурных источников, относящихся к децентрализованному определению оптимального управления многомерной динамической системой. Большинство работ по децентрализованной оптимизации, написанных за последние 15 лет, посвящены разработке и анализу различных алгоритмов метода декомпозиции-координации. Методы определения равновесных состояний по Стаккельбергу и Нэшу на сетях динамических подсистем, как обобщения классической задачи управления, получили 
развитие лишь в последние годы. За рамками данного краткого обзора остались, к сожалению, многие новые постановки задач управления второго слоя - в частности, задачи с периодической информационной структурой. Эти задачи, как и задачи децентрализованного оценивания состояния и параметров объектов управления, относящиеся к третьему слою многослойного регулятора, находятся в данное время в центре внимания исследователей.

\section{ЛИТЕ РАТ У РА}

1. Копелович А. П. Рациональная структура автоматизированной снстемы управления дискретно-непрерывным производством. - Вопр. промышл. кибернетики, вып. $35,54-56$ (1973).

2. S a nd ell, N. Jr., V a r a i ya, P., A thans, M., S a fonov, M. Survey of decentralized control methods for large-scale systems. - IEEE Trans. Automat. Contr., AC-23, № 2, 108-128 (1978)

3. Pearson, J. D. Decomposition, coordination and multilevel systems. - IEEE Trans. Syst. Sci. and Cybern., SSC-2, № 1, 36-40 (1966).

4. Findeisen, W., Pulaczewski, J., Manitius, A. Multilevel optimization and dynamic coordination of mass flow in a beet sugar plant. - Automatica, 6, № 4, 581-589 (1970).

5. al- Shaikh, A., Leffer, N. Application of multilevel control of calenders in the tire industry. - Automatica, 14, № 1, 1-18 (1978).

6. I s e rm a n n, R. On advanced methods of process computer control for industrial processes. - In: Prepr. IFAC VII Triennal World Congress, Helsinki, 1978, p. $411-421$.

7. Pradi n, B., Titli, A. Methods of decomposition-coordination for the optimization of interconnected systems, - In: Prepr. IFAC VI World Congress, part IA, Boston, 1975, paper 15. 3.

8. Soong, T. T. On model reduction of large-scale systems. - J. Math. Anal. and Appl., 60, № 2, 477-482 (1977).

9. Grujič, L. T. Singular perturbations and large-scale systems. - Int. J. Contr., 29, № 1, 159-169 (1979).

10. $\mathrm{J}$ a a ks o o, $\mathrm{O}$. Interaction analysis in multivariable control system design. - In: Prepr. II IFAC/IFIP Symp. on software for computer control, Prague, 1979, paper 22 .

11. Sil j a k, D. D. On decentralized control of large-scale systems. - In: Prepr. IFAC VII Triennal World Congress, Helsinki, 1978, p. 1849-1856.

12. Silja k, D. D. Large-scale dynamic systems : stability and structure, North Holland, Amsterdam, 1978.

13. Siljak, D. D., Vukčevic, M. B. Decentrally stabilizable linear and bilinear large-scale systems. - Int. J. Contr., 26, № 2, 289-305 (1977).

14. Mozari, M., Stephanopoulos, G., Aris, R. Finite stability regions for large-scale systems with stable and unstable subsystems. - Int. J. Contr., 26, № 5, 805-815 (1977).

15. Sinha, P. K. Controllability, observability and decoupling of multivariable systems, - Int. J. Contr., 26, № 4, 603-620 (1977).

16. Fessas, P. S. Decentralized control of linear dynamical systems via polynomial matrix methods. - Int. J. Contr., 30, № 2, 259-276 (1979).

17. Sundareshan, M. K. Exponential stabilization of large-scale systems: decentralized and multilevel schemes. - IEEE Trans. Syst. Man and Cybern., SMC-7, № 6, 478-484 (1977).

18. Sundareshan, M. K. Generation of multilevel control and estimation schemes for large-scale systems : a perturbational approach. - IEEE Trans. Syst. Man and Cybern., SMC-7, № 3, $144-152$ (1977).

19. Sundareshan, M. K. Decentral and multilevel controllability of large-scale systems. - Int. J. Contr., 30, № 1, 71-80 (1979).

20. W a ng, S. H., D a vis o n, E. D. On the stabilization of decentralized control systems. - IEEE Trans. Automat. Contr., AC-18, № 5, 473-478 (1973).

21. Gerome 1, J. C., Bernus so n, J. An algorithm for optimal decentralized regulation of linear quadratic interconnected systems. - Automatica, 15, № 4, 489491 (1979).

22. D a vison, E. The robust decentralized control of a general servomechanism problem. - IEEE Trans. Automat. Contr., AC-21, № 1, 14-24 (1976). 
23. D a rw ish, M., Soliman, H., Fantin, J. Decentralized stabilization of largescale dynamical systems. - IEEE Trans. Syst. Man and Cybern., SMC-9, № 11, 717-720 (1979).

24. A oki, M. On feedback stabilization of decentralized dynamic systems. - Automatica, 8, № 2, 163-179 (1972).

25. Sezer, M. E., H üs e y in, Ö. Stabilization of linear time invariant interconnected systems using local state feedback. - IEEE Trans. Syst. Man and Cybern., SMC-8, № 10, 751-766 (1978).

26. $M$ a h $\mathrm{moud}, \mathrm{M}$. S. A quantitive comparison between two decentralized control approaches. - Int. J. Contr., 28, № 2, 261-275 (1978).

27. Месарович М., Мако Д., Т ак ах ара И. Теория иерархических многоуровневых систем. М., «Мир», 1973.

28. Ульм С. Ю. Методы декомпозиции для решения задач оптимизации. Таллин, «валгус», 1979.

29. Handbook of large-scale systems engineering applications. Ed. by M. G. Singh and A. Titli, North Holland, Amsterdam, 1979.

30. Optimization methods for large-scale problems with applications. Ed. by A. Wismer, McGrawHill, New York, 1971.

31. Singh, M. G., D rew, A., Coales, J. Comparisons of practical hierarchical control methods for interconnected dynamical systems. - Automatica, 11, № 3, $331-340$ (1975).

32. S a nd e11, M. R., V a r a i y a, P., A than s, M. A survey of decentralized control methods for large-scale systems. - In: Proc. IFAC Symp. on LSSTA, Udine, Italy, 1976, p. 467-477.

33. M a h m o ud, M. S. Multilevel systems control and applications: a survey. - IEEE Trans. Syst. Man and Cybern., SMC-7, № 3, 125-143 (1977).

34. Athans, M. On large-scale systems and decentralized control. - IEEE Trans, Automat. Contr., AC-23, № 2, 105-106 (1978).

35. У льм С. О некоторых применениях принципа прогнозирования взаимодействий. Изв. АН ЭССР, Физ. Матем., 26, № 4, 361-368 (1977).

36. Кухтенко А. И. О теории сложных систем с иерархической структурой. В кн.: Сложные системы управления, вып. 1, Киев, «Наук. думка», 1968, c. $3-40$.

37. Поляк Б. Т. Минимизация негладких функционалов. - Ж. вычисл. матем. и матем. физ., 9, № 3, 509-521 (1969).

38. Javdan, M. A unified theory of optimal multilevel control. - Int. J. Contr., 22, № 4, 517-524 (1975).

39. M a hmoud, M., Vogt, W., Mickle, M. Multilevel control and optimization, using generalized gradients technique. - Int. J. Contr., 25, № 4, 525-543 (1977).

40. Гусев В. Б. Об одном методе декомпозиции задачи оптимального управления с линейными дифференциальными связями и смешанными ограничениями. В кн.: Актуальные вопросы теории и практики управления, М., «Наука», 1977 c. $108-113$.

41. Singh, M. G., Tit1i, A. Practical hierarchical optimization and control algorithms. - In: Prepr. IFAC VII Triennal World Congress, Helsinki, 1978, p. $1341-1352$.

42. Coh e n, G. Optimization by decomposition and coordination : a unified approach. IEEE Trans. Automat. Contr., AC-23, № 2, $222-232$ (1978).

43. W ils o n, I. D. Foundations of hierarchical control. - Int. J. Contr., 29, № 6, $899-933$ (1979).

44. Cohen, G. On an algorithm of decentralized optimal control. - J. Math. Anal. and Appl., 59, № 1, 242-259 (1977).

45. Sundareshan, $M$. Large-scale discrete systems : a two-level optimization scheme. - Int. J. Syst. Sci., 7, № 8, $901-909$ (1976).

46. S ingh, M., H a s a n, M. A comparison of two hierarchical optimization methods. - Int. J. Syst. Sci., 7, № 6, 603-611 (1976).

47. S ing h, M. G., H a s a n, M., Tit 1 i, A. Multilevel feedback control for interconnected dynamical systems using the prediction principle. - IEEE Trans. Syst. Man and Cybern., SMC-6, № 4, 233-239 (1976).

48. S ing h, M. G. A new algorithm for the one-line multilevel control of large interconnected systems with fast dynamics. - In: Prepr. IFAC/IFORS Symp. on optimization methods applied aspects, Varna, Oct. 1974 , p. $145-154$.

49. Singh, M. G., T itli, A., G a ly, J. A method for improving the efficiency of the goal coordination algorithm for large-scale dynamical systems with state variable coupling. - Comput. Elec. Eng., 2, № 4, 339-346 (1975).

50. Brosilow, C. B., Lasdon, L. S., Pearson, J. D. Feasible optimization methods for interconnected systems. - In: Proc. JACC, Troy, 1965, p. 85-92. 
51. Singh, M., Titli, A. Hierarchical feedback control for large dynamical systems. - Int. J. Syst. Sci., 8, № 1, 31-47 (1977).

52. Р а ндвеэ И. Алгоритм управления взаимосвязанными подсистемами. - Изв. АН ЭССР, Физ. Матем., 27, № 1. 4-13 (1978).

53. Malinowski, K., Ruszinski, A. Application of interaction balance methods to real process coordination. - Contr. and Cybern., 4, № 2, 15-31 (1975).

54. Michnievicz, M. Application of imbalance prediction to one-line coordination by the interaction balance method. - Arch. automat. i telemech., 23, № 4, 423-443 (1978).

55. Findeisen, W., Malinowski, K. Two-level control and coordination for dynamical systems. - Arch. automat. i telemech., 24, № 1, 3-27 (1979).

56. Joall a nd, G., Cohen, G. Optimal control of a water distribution network by various multilevel methods. - In: Prepr. IFAC VII Triennal World Congress, Helsinki, 1978, p. $1429-1436$.

57. al-S ha ikh, A., T u, F. Multilevel control of continuous digesters. Applications and results. - In: Prepr. IFAC VII Triennal World Congress, Helsinki, 1978, p. $239-248$.

58. H a s s a n, M., Hute a u, R., S ing h, M., T it $1 \mathrm{i}, \mathrm{A}$. A three-level constate prediction method for continuous dynamical systems. - Automatica, 14, № 2, 177-181 (1978).

59. S ingh, M. G., H a s a n, M. A two-level prediction algorithm for nonlinear systems. - Automatica, 13, № 1, 95-98 (1977).

60. M a h moud, M. S. Optimal control of constrained problems by the costate coordination structure. - Automatica, 14, № 1, 31-41 (1978).

61. S in $\mathrm{g} \mathrm{h,} \mathrm{M.,} \mathrm{H} \mathrm{a} \mathrm{s} \mathrm{s} \mathrm{a} \mathrm{n,} \mathrm{M.} \mathrm{Hierarchical} \mathrm{optimization} \mathrm{for} \mathrm{non-linear} \mathrm{dynamic} \mathrm{systems}$ with non-separable cost functions. - Automatica, 14, № 1, 99-101 (1978).

62. Nishikawa, Y., Oijka, T., Shimazutsu, H., Okudaira, M. An interaction coordination algorithm with modified performance index for nonlinear optimal control problems. - J. Optimiz. Theory Appl., 25, № 2, 229-245 (1978).

63. Is himatsu, T., Mohri, A, Takat a, M. Optimization of weakly coupled subsystems by two-level method. - Int. J. Contr., 22, № 6, 877-882 (1975).

64. Бр усил овский А. М., Островски й Г. М. Об одном декомпозиционном методе оптимизашии сложных систем. - Изв. АН СССР, Техн. кибернетика, № 3, $17-24$ (1978).

65. Grate 1 o u p, G., T it li, A. Two-level dynamic optimization methods. - J. Optimiz. Theory Appl., 15, № 5, 533-547 (1975).

66. J a v d a n, M. R., Ri chards, R. J. Decentralized control systems theory. A critical evaluation. - Int. J. Contr., 26, № 1, 129-144 (1977).

67. J a v d a n, M. R. Extension of dual coordination to a class of nonlinear systems. Int. J. Contr., 24, № 4, 551-571 (1976).

68. S in g h, M. G., T a m u r a, H. Modeling and hierarhical optimization for oversaturated urban road traffic networks. - Int. J. Contr., 20, № 6, 913-934 (1974).

69. S to ilov, E. Method of augmented Lagrangians in two-level static optimization. Arch. automat. i telemech., 22, № 2, 219-222 (1977).

70. Macko, D., Haimes, Y. Overlapping coordination of hierarchical structures. IEEE Trans. Syst. Man and Cybern., SMC-8, № 10, 745-750 (1978).

71. Бойчук Л. М. Методы многоуровневого управления взаимосвязанными статическими объектами. - Автоматика, № 4, 52-59 (1979).

72. Wat anabe, N., Nishimura, Y., Matsubara, M. Decomposition in largesystem optimization using the method of multipliers. - J. Optimiz. Theory Appl., 25, № 2, 181-193 (1978).

73. Murtagh, B. A., S a nders, M. A. Large-scale linearly constrained optimization. - Math. programming, 14, № 1, 41-72 (1978).

74. Simmons, M. D., White, W. T. The decentralized profit maximization of interconnected production systems, - Int. J. Contr., 25, № 5, 705-719 (1977).

75. У льм С. Ю. О некоторых методах иерархической оптимизации и агрегации. В кн.: Проблемы планирования и управления экономическими целенаправленными системами. Новосибирск, «Наука», 1972, с. ๔4-78.

76. Первозванский А. А., Гайцгори В. Г. Декомпозиция, агрегирование и приближенная оптимизащия. М., «Наука», 1979.

77. Medanic, J., Radojevic, D. On the multilevel Stackelberg strategies in linear quadratic systems. - J. Optimiz. Theory Appl., 24, № 3, 485-497 (1978).

78. Medanic, J. Closed loop Stackelberg strategies in linear-quadratic problems. In: Proc. JACC, 1977, San Francisco, 1977, p. 1324-1329.

79. Cruz, J. B. Jr. Leader-follower strategies for multilevel systems. - IEEE Trans. Automat. Contr., AC-23, № 2, 244-255 (1978).

80. Ga rdner, B. F. Jr, Cruz, J. B. Jr. Feedback Stackelberg strategy for multilevel 
hierarchical games. - IEEE Trans. Automat. Contr., AC-23, № 3, 489-491 (1978).

81. К расовский Н. Н. Игровые задачи управления. - Тр. Ин-та матем. и мех. У ральского НЦ АН СССР, 1977, с. 32-45, 115-116.

82. Понтрягин Л. С. Оптимизация и дифференциальные игры. - Успехи матем. наук, 33, № 6, 27-28 (1978).

83. P a pavassilopoulos, G. P., Cruz, J. B. Jr. Nonclassical control problems and Stackelberg games. - IEEE Trans. Automat. Contr., AC-24, № 2, 155-165 (1979).

84. S t a r r, A. W., H o, Y. C. Nonzero-sum differential games. - J. Optimiz. Theory Appl., 3, № 2, 184-219 (1969).

85. Simaan, M., Cruz, J. B. Jr. On the Stackelberg strategy in nonzero-sum games. - J. Optimiz. Theory Appl., 11, № 5, 533-555 (1973).

86. B a sar, T. On the relative leadership property of Stackelberg strategies. J. Optimiz. Theory Appl., 11, № 6, 655-661 (1973).

87. H o, Y. Differential games, dynamic optimization and generalized control theory. J. Optimiz. Theory Appl., 6, № 3, 179-209 (1970).

88. Ho, Y. Decisions, control and extensive games. - In: Proc. IFAC VI Congress, Boston, USA, 1975, paper 16.1 .

89. B a s a r, T., S elbuz, H. Properties of Nash solutions of a two-stage nonzero-sum games. - IEEE Trans. Automat. Contr., AC-21, № 1, 48-54 (1976).

90. B a sa r, T. Nash strategies for $M$-person differential games with mixed iniormational structures. - Automatica, 11, № 4, 547-551 (1975).

91. Hämelä in en, R. P. Recursive algorithm for Nash strategies in two-player differential games. - Int. J. Contr., 27, № 2, 220-237 (1978)

92. B a s a r, T. Informationally nonunique equilibrium solutions in differential games. SIAM J. Contr. and Opt., 15, № 4, 636-660 (1977).

93. O z g ü n e r, U., P e r kin s, W. Optimal control of multilevel large-scale systems. Int. J. Contr., 28, № 6, $967-980$ (1978).

94. Ozgüner, Ü., Perkins, W. A Nash feedback approach to control of river pollution. - IEEE Trans. Syst. Man and Cybern., SMC-9, № 5, 290-294 (1979).

95. Breton, A., Haurie, A., Kalocsai, R. Efficient management of interconnected power systems: a game theoretic approach. - Automatica, 14, № 5, 443-453 (1978).
Ннститут кибернетики
Академии наук Эстонской ССР
Поступила в редакцию 3/VII 1980

\section{RANDVEE}

\section{ULEVAADE JUHTIMISULESANDE DETSENTRALISEERIMISMEETODITEST}

On esitatud lühiülevaade artiklitest ja konverentsiettekannetest, mis käsitlevad mitmekihilise juhtimissüsteemi $\left[{ }^{1-7}\right]$ teise kihi juhtimisülesande (JU) kolme pōhilise variandi detsentraliseeritud lahendusskeeme. Seostatud alamobjektide vôrgu lokaalsete sihifunktsioonide summa minimeerimise probleeme (JU 1. variant) on viimase 15 aasta jooksul pōhjalikult uuritud $\left[{ }^{27-76}\right]$. Oha suurenevat tähelepanu leiavad meetodid, mida rakendatakse lokaalsete sihifunktsioonide minimeerimiseks etteantud järjestuses dünaamiliste alamobjektide üldisel $\left[{ }^{77-86}\right]$ (JU 2. variant) ja atsüklilisel vôrgul $\left.{ }^{87-95}\right]$ (JU 3. variant).

\section{RANDVEE}

\section{SURVEY OF DECENTRALIZED CONTROL METHODS}

This paper surveys the contributions which handle the control problem of the second layer of symbolic multilayer control structure $\left[{ }^{1-7}\right]$ based on the multilevel approach. Restricting ourselves to the work that has been contributed through the deterministic 
46

И. Рандвеэ

linear-quadratic dynamic optimization problems, attention has been paid to structural issues.

A large-scale composite system was modelled as an interconnection of subsystems. A local cost function was associated with each of them and a control problem based on three different solution concepts was stated. Minimization of the sum of local cost function has lead to a well-known two-level decomposition-coordination algorithms $\left[{ }^{27-76}\right]$. The arrangement of the subsystems in a linear hierarchy of decision making resulted in a multilevel Stackelberg strategy ${ }^{[7-86}$. The Nash solution is apparent when loop-free interconnection structure is adopted [87-95].

This unification, supported by the short list of papers on the control problem of the first layer- on decentralized feedback control $\left[{ }^{8-26}\right]$ - enables to cover a substantial portion of the control theoretic literature on decentralized and hierarchical systems.

It should be pointed out that there is a growing interest in the analysis and practical application of a much larger class of systems than has been covered by this short survey. Much work has been done in recent years in an attempt to generalize the optimail control theory towards many-person dynamic optimization, on decentralized state estimation and parameter identification. Optimization problems have been so far found to be most tractable for decentralization. The techniques developed, however, are effecfive for linear systems with linear interconnection equations as well as for a certain class of non-linear systems. A larger class of non-linear systems need to be covered for the approach to have a greater practical significance. 